\title{
Mixed particle beam for simultaneous treatment and online range verification in carbon ion therapy: Proof-of-concept study
}

\author{
Davide Mazzucconia) \\ Energy Department, Politecnico di Milano, via Lambruschini 4, Milano 20156, Italy \\ Fondazione CNAO Strada Privata Campeggi, Pavia 27100, Italy \\ Stefano Agosteo \\ Energy Department, Politecnico di Milano, via Lambruschini 4, Milano 20156, Italy \\ Michele Ferrarini \\ Fondazione CNAO Strada Privata Campeggi, Pavia 27100, Italy \\ Luigi Fontana \\ Elitalia srl, Via Grossich, 32, Milano 20131, Italy
}

Valeria Lante, Marco Pullia, and Simone Savazzi

Fondazione CNAO Strada Privata Campeggi, Pavia 27100, Italy

Purpose: Radiation therapy with ion beams provides a better conformation and effectiveness of the dose delivered to the tumor with respect to photon beams. This implies that a small uncertainty or variation in the crossed tissue shape and density may lead to a more important underdosage of the tumor and/or an overdosage of the surrounding healthy tissue. Although the online control of beam fluence and transverse position is well managed by an appropriate beam delivery system, the online measurement of the longitudinal position of the Bragg peak inside the patient is still an open issue. In this paper we propose a proof-of-concept study of a technique that would allow the online verification of the patient thickness along the beam direction, which could permit detecting a subset of possible range error causes, such as morphological variations.

Methods: The nuclei ${ }^{12} \mathrm{C}$ and ${ }^{4} \mathrm{He}$ have the same magnetic rigidity: the two species could be accelerated together in an accelerator and a mixed particle beam delivered to the patient. In the same medium and with the same energy per nucleon, the range of ${ }^{4} \mathrm{He}^{2+}$ is about three times the ${ }^{12} \mathrm{C}^{6+}$ one. It is, thus, conceivable to achieve a dual goal with a single mixed beam: carbon, stopping into the tumor, is appointed to cure, while helium, emerging from the patient, to control: by detecting and measuring the residual range and position of $\mathrm{He}$, it would be possible to determine the integrated relative stopping power of the patient and prove that it is the expected one. For the detection of helium particles, a plastic scintillator and an optical sensor are proposed. Being helium ions not available at $\mathrm{CNAO}$, the detection system has been characterized using a proton beam. Nevertheless, since the light emitted by a proton is less than the one produced by a helium ion, the helium signal is expected to be more pronounced than the proton one (for the same number of particles). To predict the magnitude of the light signal measured by the sensor, two Monte Carlo models have been setup and validated by measuring the photons per pixel impinging on the sensor. To deal with the many optical issues and to reliably describe the physical process, some corrections have been included into the models.

Results: The predictions of both the models are in good agreement with the measurements (within the $20 \%$ in terms of absolute photons per pixel). The proposed detection system is able to measure the range of a proton beam with sub-millimetrical precision also in the presence of the background produced by carbon ion fragments and discrepancies in the expected range were detected with a resolution better than $1 \mathrm{~mm}$.

Conclusions: Although many technical issues have still to be addressed for a real implementation in a clinical environment, the preliminary results of this study suggest that a surrogate of real-time verification of the beam range inside the patient during a treatment with carbon ions is possible by adding a small fraction of helium ions to the primary beam.

Key words: FLUKA, hadrontherapy, helium, plastic scintillator 


\section{INTRODUCTION}

In the last decades, radiation therapy with ion beams has been spreading worldwide for the treatment of cancer. With respect to conventional photon radiation therapy, better dose conformation to the target and increased sparing of the healthy tissue surrounding the tumor can be achieved with ion beams, due to the different physics of interaction of charged particles into matter. Moreover, higher LET (linear energy transfer) radiation, like carbon ions, exhibits a larger number of double strand breaks in the DNA and a higher RBE in the peak than photons and protons and is, thus, a good candidate for the treatment of radio-resistant tumors. ${ }^{1}$

Better conformation and effectiveness imply that any uncertainty in the crossed tissue density and shape may lead to a severe underdosage of the tumor and/or an overdosage of the surrounding healthy tissue, making ion beam therapy highly sensitive to any morphological patient variation. Therefore, it is mandatory to ensure a precise knowledge of the patient's internal anatomical conformation. ${ }^{2}$ Besides, during the delivery of fractionated radiotherapy, range deviations may occur because of minor inaccuracies in patient positioning or morphological changes, leading to local density modifications with respect to the planning $\mathrm{CT}^{3}$

Many efforts are nowadays devoted to the investigation of various techniques in order to verify the ion beam range inside the patient, such as in-beam positron emission tomography (PET), prompt particles detection (neutral or charged) or ion-based imaging. The first has already been implemented in clinical environment and is very promising, although it suffers from the drawback of intrinsically low signal and the issue of biological washout. ${ }^{4-6}$ The second exploits the production of secondary charged and neutral particles by the primary ion beam, whose detection can be used for an online check of the beam particle range. Techniques based on the detection of secondary prompt photons are recently starting a promising clinical experimentation and they are mainly focused on proton therapy. ${ }^{7}$ On the other hand, charged particles are being studied for ion therapy showing preliminary promising performances. ${ }^{8}$ The latter uses the same source employed for the treatment, with the purpose of obtaining a radiography of the patient just before the tumor irradiation: the beam should be energetic enough to pass through the body and reach a measuring system. A plastic scintillator, coupled to a CCD camera, or a stack of scintillators are typically employed coupled with other detectors making possible the range measurement for each particle. Ion-based radiography has the advantage to be available directly in the treatment room, for the verification of range and also patient anatomy. The main limitations of this technique are the need of a machine able to accelerate to energies higher than the clinical ones, the intrinsic impossibility of realtime monitoring, the low possible fluences, to limit the additional dose to the patient, and the unsatisfactory speed of the state of the art detectors, unable to provide a liveresult of the radiography. ${ }^{9}$
In this paper, we propose a proof-of-concept study of a new technique capable to detect, in real time, density modifications, with respect to the planning $\mathrm{CT}$, in the tumor target due to morphological changes or minor inaccuracies in patient positioning. The idea is to add a small fraction of ${ }^{4} \mathrm{He}^{2+}$ to the primary ${ }^{12} \mathrm{C}^{6+}$ beam, to accelerate and deliver the two species together: while carbon ions are deputed to cure, helium is appointed to verify the patient thickness along the beam direction, thanks to its larger range.

We also propose a measurement system based on a scintillator and an optical sensor and we also show the performances of this system in evaluating the residual beam range. For reasons that will be better specified in the text, the detection system has been characterized using a proton beam instead of helium.

\section{MATERIALS AND METHODS}

\section{A. Mixed beam}

Ions ${ }^{4} \mathrm{He}^{2+}$ and ${ }^{12} \mathrm{C}^{6+}$, with the same momentum per nucleon, have similar magnetic rigidity, having rest mass to charge ratio 4.00236/2 a.m.u. and 12/6 a.m.u., respectively. This peculiarity allows to accelerate in a synchrotron carbon and helium at the same time and to irradiate the patient with a mixed beam, where the two species have the same kinetic energy per nucleon. The energy loss of carbon and helium ions is different: in the same medium and at the same velocity, the CSDA (Continuous Slowing Down Approximation) range of helium is approximately three times the carbon's one. ${ }^{10}$ Therefore, by adding a little fraction of helium to the carbon beam, it would be viable to deliver the conventional therapeutic dose with C-ions and, simultaneously, to perform a real-time verification of the patient thickness/position with the emerging helium ions, as schematically illustrated in Fig. 1.

In fact, by measuring the residual range of the helium particles reaching a detector located downstream the target, it would be feasible to monitor that the integrated density and/ or the thickness of the patient is the expected one.

At CNAO, an active scanning system is employed to give the dose to the patient: the beam is delivered to the target volume using two scanning magnets. If a mixed beam is scanned on the patient, the helium residual range could be detected in correspondence of each scanned position and, thus, a map of

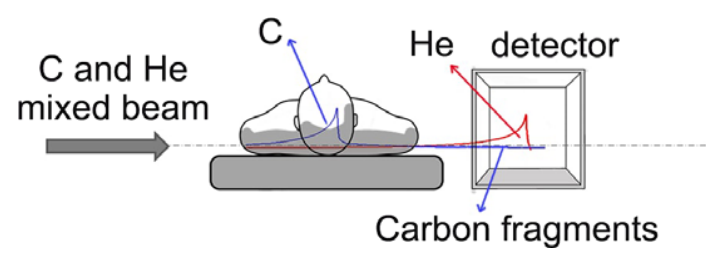

FIG. 1. Conceptual scheme of the system (Bragg peaks for helium and carbon are not to scale). Carbon stops into the patient delivering the dose, while helium is measured by the detector. [Color figure can be viewed at wileyon linelibrary.com] 
helium residual range could be reconstructed for the whole treated volume. This map could be very useful for a real-time check of the correctness of the patient position and/or shape. It is important to notice that the detector must be able to measure helium residual range in the presence of secondary carbon fragments that, unavoidably, impinge on the detector and create an unwanted signal.

Thus, the fraction of $\mathrm{He}$ ions to be introduced has to be large enough to be visible above the background but small enough for its contribution to the total dose not to be harmful.

Since the energy loss scales with $\mathrm{Z}^{2}$ and the charge of $\mathrm{He}$ is one-third of the charge of $\mathrm{C}$, each $\mathrm{He}$ ion deposits approximately $1 / 10$ of the dose with respect to a C-ion. Adding $10 \%$ of $\mathrm{He}$ ions to the $\mathrm{C}$ beam shall result in approximately $1 \%$ dose increase, which we consider to be acceptable because it is of the same order of magnitude of the tolerance on the dose accuracy and will anyway be considered during the treatment planning phase to be finally accepted by the physician responsible of the treatment.

For a proper verification a Monte Carlo simulation, using FLUKA, ${ }^{11,12}$ of a representative carbon ion treatment plan performed at CNAO has been carried out in which, to each carbon beam that builds the spread out Bragg peak (SOBP), a $10 \%$ in number of ${ }^{4} \mathrm{He}$ particles was added.

The absorbed doses of carbon and helium ions are shown in Fig. 2. The sum of proximal doses of helium is represented by the red line (close to the $\mathrm{x}$ axis) in the figure: it amounts to an additional absorbed dose of about $20 \mathrm{mGy}$, with respect to the 1.8 Gy given by carbon particles in correspondence of the SOBP. Since the additional dose given by helium particles is around $1 \%$ of the SOBP, much lower than the proximal dose of C-ions and the tail due to the fragments, and since the He peak is sufficiently visible, as will be shown later, in all the following evaluations the ratio, in number, between the two species has been kept equal to $10 \%$.

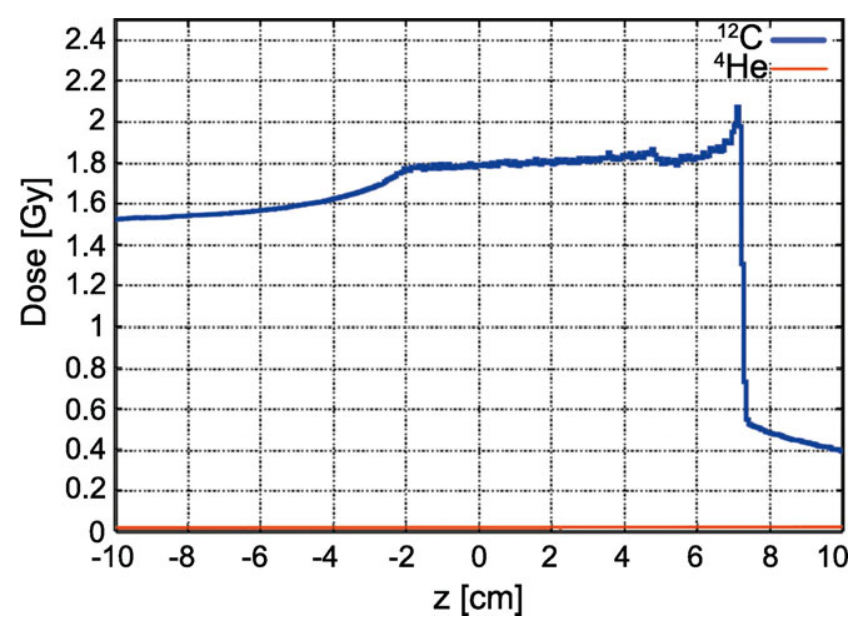

FIG. 2. FLUKA simulation of helium and carbon dose comparison. To each carbon beam that builds the spread out Bragg peak (SOBP), a $10 \%$ in number of ${ }^{4} \mathrm{He}$ particles was added. [Color figure can be viewed at wileyonlinelibra ry.com]

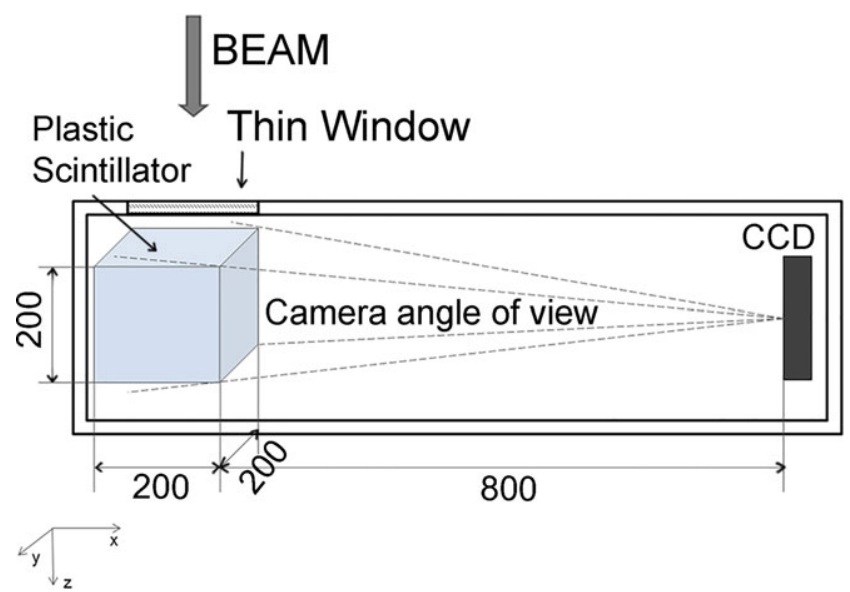

FIG. 3. Experimental setup for the measure of the particle range. The measuring system prototype is composed of a plastic scintillator observed by CCD camera, with a resolution of $4008 \times 2672$ pixels and an image area of $36 \mathrm{~mm} \times 24.7 \mathrm{~mm}$, mounting a Nikkor lens. The detector is a cubic polyviniltoluene-based scintillator of $20 \mathrm{~cm}$ lateral dimension. [Color figure can be viewed at wileyonlinelibrary.com]

\section{B. Experimental setup}

The typical intensities of $\mathrm{C}$-ions employed in clinical practice and, therefore, the expected fluences of ${ }^{4} \mathrm{He}$ ions drove the choice of the detector.

At CNAO, the beam is delivered in spills lasting about $1 \mathrm{~s}$ and with a maximum number of particles of about $4 \times 10^{8}$. The typical dose deposited in a tumor in a single fraction is about 2 Gy, corresponding to about $10^{9}$ particles in the distal slice, for a slice of dimensions $100 \times 100 \times 3 \mathrm{~mm}^{3} \cdot{ }^{13}$ Assuming the slice to be subdivided in $33 \times 33$ voxels with a volume of $3 \times 3 \times 3 \mathrm{~mm}^{3}$ and a uniform dose distribution, the number of carbon ions delivered in each voxel is about $9.5 \times 10^{5}$. In a single spill, about 420 voxels $\left(4 \times 10^{8} / 9.5 \times 10^{5}\right)$ are treated in about $2 \mathrm{~ms}$ each.

In the hypothesis of adding a $10 \%$ in number of ${ }^{4} \mathrm{He}$ particles to the primary beam, $9.5 \times 10^{4}$ helium ions are delivered to each voxel. This corresponds to a fluence rate of helium at the patient entrance of the order of $4.5 \times 10^{8}$ part/ $\left(\mathrm{cm}^{2} \mathrm{~s}\right)^{\mathrm{i}}$.

In addition, a large number of fragments, produced by the interaction of the primary beam with the patient, impinges on the detector. The exact number depends on the particle energy and on the target thickness; a graphical evaluation is given by the tail after the SOBP in Fig. 2 .

The measuring system prototype, represented in Fig. 3, is composed of a plastic scintillator observed by CCD camera (SBIG STL-11000M), with a resolution of $4008 \times 2672$ pixels and an image area of $36 \mathrm{~mm} \times 24.7 \mathrm{~mm}$, mounting a Nikkor $85 \mathrm{~mm}$ F/1.4 lens. The detector is a cubic polyviniltoluene based scintillator (Saint Gobain Crystals BC-408) of $20 \mathrm{~cm}$ lateral dimension, emitting light at $425 \mathrm{~nm}$.

The preliminary evaluation of the feasibility of such a detection system for the residual range was performed at

${ }^{\mathrm{i}}$ Fluence rate estimation: $9.5 \times 10^{4} /\left(9 \times 10^{-2} \mathrm{~cm}^{2} \times 1 \mathrm{~s} / 420\right)$. 
CNAO using protons and carbon ions, since helium is not yet available.

Moreover because of the low current of $\mathrm{C}^{6+}$ provided by the CNAO sources, $\mathrm{C}^{3+}$ together with $\mathrm{He}^{+}$should be used, but unluckily the CNAO linac cannot accelerate ions with $\mathrm{A} /$ $\mathrm{Q}>3$.

Being the light emitted by a proton less than the one produced by a helium ion (lower stopping power of protons), the helium Bragg peak is expected to be more clearly distinguishable from fragments than the proton one (for the same number of particles). In order to have a conservative characterization of the detector, from the light emission point of view, the employed number of protons has been kept the same foreseen for helium ions (10\% in number).

The main idea is to take an image of the scintillation light of the scintillator and use this image to evaluate the proton (and helium in future) residual range. ${ }^{14,15}$

The CCD sensor is at a distance of $80 \mathrm{~cm}$ and all the system is placed in a closed box in order to avoid any external light interference. The thin window in correspondence with the scintillator assures low beam degradation during the irradiation.

The foremost purpose of the measurements is the validation of two Monte Carlo models conceived to evaluate the scintillator quenching coefficient and to compare measured and simulated number of photons per pixel detected by the CCD sensor. In this way, knowing the quantum efficiency of the sensor and the gain, in terms of electrons per logical unit, a "model" image could be built in order to foresee the digital one.

\section{C. Monte Carlo models}

The two Monte Carlo models are both based on FLUKA and differ, mainly, in the evaluation of the number of photons per pixel reaching the CCD sensor.

They have in common the Monte Carlo simulation of the dose deposition inside the scintillator but they differ in the method by which the emitted light is transported from the emission point to the CCD sensor.

The first model is purely numerical and exploits the FLUKA capability in generating and propagating (according to the geometrical optics laws) optical photons.

The optical photons are generated in the plastic scintillator by the primary particle; they are transported (by the code) up to the interface between the plastic scintillator and the air and, finally, only those that can reach the lens aperture and the sensor (i.e., emerging within the angle $\vartheta_{\max }$ in Fig. 4) are selected. The selection of these photons is determined by: the ratio of the refraction indexes of air and plastic scintillator (1 and 1.58 , respectively), the focal length of the optics, the value of the diaphragm and the distance between the scintillator and the sensor $(80 \mathrm{~cm})$.

The second model is semianalytical: it uses FLUKA only to calculate the energy deposited into the scintillator, while both the production and the propagation of the optical photons are carried out analytically, according to the laws of geometric optics. As illustrated in Fig. 4, the scintillator has length $\mathrm{L}_{\mathrm{s}}$ and is centered in the origin of the reference frame.

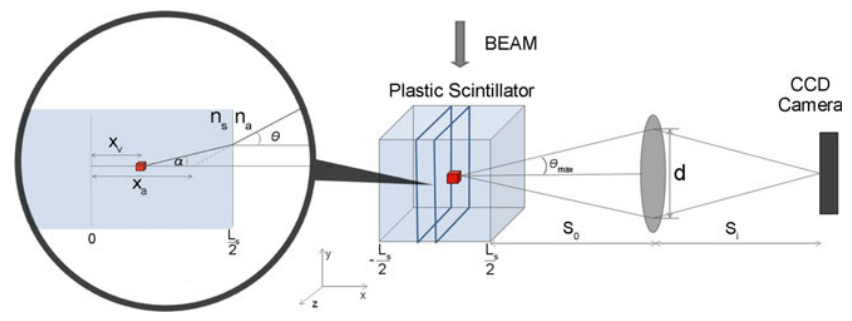

FIG. 4. Schematic representation of the optical system. The small cube represents a single 3D pixel. The inset is meant to illustrate refraction artifacts. Refer to the text for the details of the Monte Carlo model. [Color figure can be viewed at wileyonlinelibrary.com]

The distances between the scintillator and the lens and the lens and the CCD sensor are $S_{o}$ and $S_{i}$, respectively. The scintillator is divided in a 3D pixel matrix: the red cube in the figure represents a single $3 \mathrm{D}$ pixel. Its center has coordinates $(\mathrm{x}$, $\mathrm{y}, \mathrm{z})$ and its distance with the lens is $\mathrm{S}_{\mathrm{o}}+\frac{\mathrm{L}_{\mathrm{s}}}{2}-\mathrm{x}$. The depth of a single pixel is $\Delta \mathrm{x}$. The photons produced in the scintillator and impinging the CCD sensor are those emerging within the solid angle $\vartheta_{\max }$. Because of refraction, the corresponding limit angle within the scintillator, $\alpha_{\max }$, is smaller, as illustrated in the inset. Furthermore, the image recorded by the CCD camera would seem to be produced closer to the scintillator edge, i.e., at a distance $\mathrm{x}_{\mathrm{a}}$. The FLUKA output is the energy deposited by the beam into each $3 \mathrm{D}$ pixel. The intensity of the signal produced by a single pixel of the CCD sensor depends mainly on the number of scintillation photons produced, the lens coupling efficiency and the efficiency in converting the photon in the digital gray scale.

Let us consider the light in the scintillator produced on a plane of depth $\Delta x$ located at a distance $S_{0}+\frac{L_{s}}{2}-x$ from the lens. The whole signal at the CCD sensor is the linear combination of the contribution of each plane, neglecting attenuation, refraction and focusing ${ }^{\text {ii }}$.

The number of photons $\mathrm{N}_{\gamma}$ produced in each pixel of the scintillator is given by:

$$
\mathrm{N}_{\gamma}=\mathrm{D}(\mathrm{x}, \mathrm{y}, \mathrm{z}) \cdot \mathrm{A}_{\mathrm{p}} \cdot \Delta \mathrm{x} \cdot \rho \cdot \mathrm{S} \cdot \mathrm{Q}
$$

$\mathrm{D}(\mathrm{x}, \mathrm{y}, \mathrm{z})$ is the dose released, as simulated by FLUKA, in the 3D scintillator pixel, centered in $(x, y, z)$ and of depth $\Delta x . A_{p}$ is the area of the pixel as seen by the sensor, $\rho$ is the scintillator density and $\mathrm{S}$ the scintillator light yield. $\mathrm{Q}$ is a quenching factor and is related to the Birks coefficient. ${ }^{10} \mathrm{~A}_{\mathrm{p}}$ is related to the characteristic dimension of a pixel of the sensor $h_{i}$, through the magnification ratio $\mathrm{m}(\mathrm{x}): \mathrm{A}_{\mathrm{p}}=\left(\mathrm{h}_{\mathrm{i}} / \mathrm{m}(\mathrm{x})\right)^{2}$. Neglecting refraction, $m(x)=S_{i} \cdot\left(S_{o}+\frac{L_{s}}{2}-x\right)^{-1}$, where $S_{i}$ and $S_{0}+\frac{L_{s}}{2}-x$ are the distances between the lens and the CCD and the lens and the scintillator pixel, along the optical axis, respectively, as illustrated in Fig. 5.

Assuming that each voxel in the scintillator is a source of isotropic light, it is possible to define a lens coupling efficiency, as it is more precisely described in literature ${ }^{14-17}$ :

\footnotetext{
${ }^{i i}$ The lens diaphragm was set small enough to have the entire scintillator depth on focus. This approximation allows the use of the thin lens law for each $\mathrm{x}$.
} 


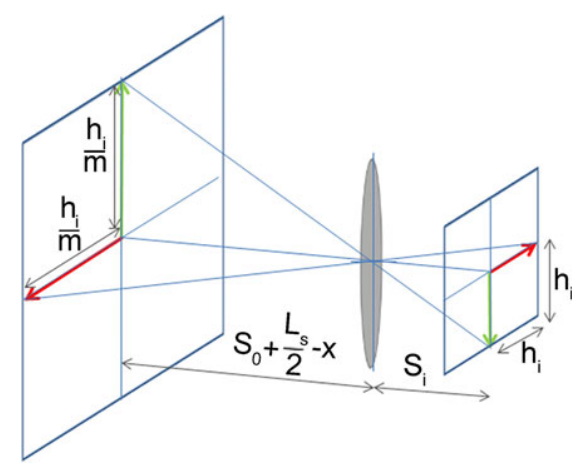

FIG. 5. Taking as a reference the size of a sensor pixel $h_{i}$ (right in the schematic view), the edge of the 3D pixel in the scintillator is $h_{i} / m$, where $m$ is the magnification ratio. This last varies with the distance of the scintillator pixel from the lens. [Color figure can be viewed at wileyonlinelibrary.com]

$$
\varepsilon=T \frac{\int_{0}^{2 \pi} \int_{0}^{\alpha_{\max }} \sin \vartheta d \vartheta d \varphi}{\int_{0}^{2 \pi} \int_{0}^{\pi} \sin \vartheta d \vartheta d \varphi}=T \sin ^{2}\left(\frac{\alpha_{\max }}{2}\right)
$$

where $\mathrm{T}$ is the lens transmission coefficient and $\alpha_{\max }$ describes the cone by which an emitted photon impinges the CCD sensor, as illustrated in Fig. 4. The corresponding limit angle in air, $\vartheta_{\max }$, can be evaluated through the Snell law:

$$
n_{s} \sin \alpha_{\max }=n_{a} \sin \vartheta_{\max }
$$

where $n_{s}$ and $n_{a}$ are the refraction indexes in the scintillator and in air, respectively. If $\vartheta_{\max }$ is small enough, with some manipulations, it can be written:

$$
\varepsilon=T\left(\frac{d n_{a}}{4 n_{s} S_{o}}\right)^{2}
$$

$\mathrm{d}$ is the diameter described by the diaphragm aperture, i.e., $d=f / F$, where $f$ is the focal length and $F$ is the diaphragm aperture. Applying the thin lens law $S_{o}^{-1}+S_{i}^{-1}=f^{-1}$, the lens coupling efficiency can be expressed in terms of the magnification ratio $\mathrm{m}\left(\mathrm{m}=\mathrm{S}_{\mathrm{i}} / \mathrm{S}_{\mathrm{o}}\right)$

$$
\varepsilon=\frac{T}{16 F^{2} n_{s}^{2}} \cdot \frac{m^{2}}{(1+m)^{2}}
$$

In the 3D case, the magnification ratio and thus the lens coupling efficiency depend on the depth of the focused pixel inside the scintillator ( $\mathrm{x}$ coordinate).

Finally, the overall calculated light impinging on the sensor is obtained by integrating the signal coming from each plane of the same lens coupling efficiency (same $\mathrm{x}$ in the equation, i.e., same distance between the sensor and the considered point) over the scintillator width, ${ }^{15}$ according to:

$$
\begin{aligned}
I= & \int_{\frac{-L_{s}}{2}}^{\frac{-L_{s}}{2}} D(x, y, z) \cdot\left(\frac{h_{i}}{m(x)}\right)^{2} \cdot \rho \cdot S \cdot Q \cdot \frac{T}{16 F^{2} n_{s}^{2}} \\
& \cdot \frac{m^{2}(x)}{(1+m(x))^{2}} \cdot d x
\end{aligned}
$$

The integration neglects parallaxes and is performed numerically.
Both Monte Carlo models can give, as an output, a simulated 2D image acquired by the sensor, but, for all the subsequent comparisons, the light is further integrated along the direction perpendicular to the beam direction (in order to have a 1D distribution for an easier comparison).

To test the Monte Carlo models in the presence of carbon fragments, a two-steps scheme has been used. Since a mixed proton and carbon beam cannot be accelerated (because of different $A / Q$ ), the total signal is obtained by numerically summing up two separated digital images.

A first test session (max available proton energy, $32 \mathrm{~cm}$ range) was devoted to identification of the Bragg peak above the background. In this session the first image was made with a $20 \mathrm{~cm}$ water slab placed upstream the scintillator to create fragments with a carbon ion beam. The second image was obtained with the same geometry with a proton beam. The number of primary and secondary particles was chosen to be $5 \times 10^{6}$ and $5 \times 10^{5}$, respectively, while the full width half maximum at the isocenter in air was $6 \mathrm{~mm}$ for carbon ions and $10 \mathrm{~mm}$ for protons and the energy of protons and carbon ions was 226.9 and $226.5 \mathrm{MeV}$ and $\mathrm{MeV} / \mathrm{u}$, respectively (the expected range in water is 32.2 and $10.7 \mathrm{~cm}$, respectively). The number of particles was chosen as low as possible while still being measurable by the CNAO dose delivery system.

\section{D. Setup for the beam range monitoring}

A second test session was setup in order to test the capability in determining the Bragg peak position (and the residual particle range) even when the beam is scanned on the treated volume, the scintillator has been irradiated in nine different positions $50 \mathrm{~mm}$ aside from each other, forming a nine points square centered in the scintillator $\mathrm{x}, \mathrm{y}$ face.

In this and in the following test sessions, an alternative sensor (ZWO ASI $174 \mathrm{MM}$ with a resolution if $1936 \times 1216$ pixels) and a different focal length matching the new sensor have been used. The reason for the new setup was to test an alternative cheaper sensor able in having a depth of field greater than $20 \mathrm{~cm}$ (i.e., scintillator lateral dimension). The drawback is a lower sensitivity which required a larger number of particles. The scintillator was irradiated with $81 \mathrm{MeV}$ protons $\left(5 \times 10^{6}\right.$ per spot) and with $172.4 \mathrm{MeV} / \mathrm{u}$ carbon ions $\left(5 \times 10^{7}\right.$ per spot). During the irradiation with $\mathrm{C}$-ions a water equivalent thickness of $15 \mathrm{~cm}$ was positioned in front of the detector to produce fragments. $172.4 \mathrm{MeV}$ corresponds to the energy protons should have to exit from the water equivalent thickness in front of the detector with energy of $81 \mathrm{MeV}$. In this measurement the $15 \mathrm{~cm}$ slab was not in place when protons were used because protons scatter more than helium and the spot would have been larger than needed. In the real case, He should traverse the slab and part of it would be lost in nuclear interactions, but the surviving fraction exceeds $70 \%$ and thus the simulation with protons is still conservative. The fragments created by $\mathrm{He}$ are negligible with respect to those created by carbon.

The intensities of the scintillation light are acquired superimposing protons and carbon ions for different $(\mathrm{x}, \mathrm{y})$ positions 


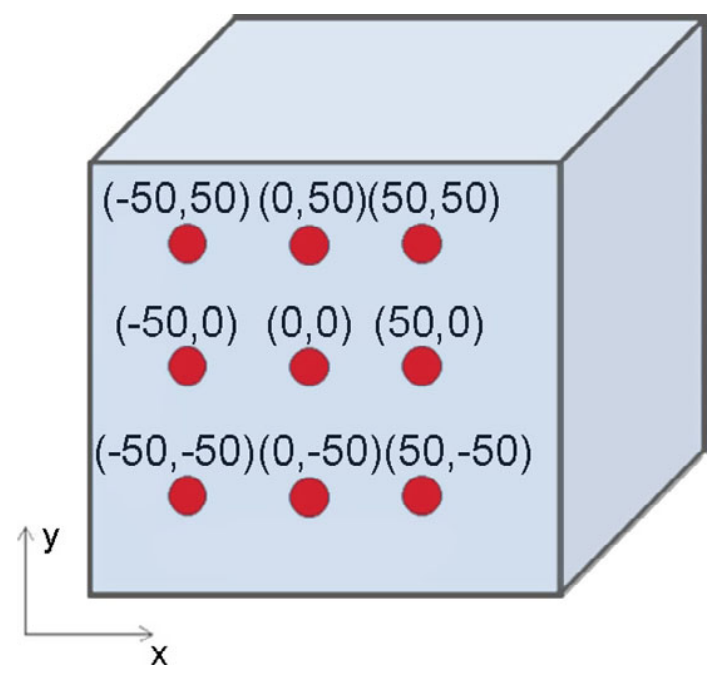

FIG. 6. Irradiated points on the scintillator for particle range measurement. The beam follows $\mathrm{z}$ direction and the optical sensor is in the positive $\mathrm{x}$ direction. [Color figure can be viewed at wileyonlinelibrary.com]

in the scintillator and summing up the two separated digital images, as explained before.

Finally, to verify the capability of the system to detect a range variation of the incoming particles, a monoenergetic proton beam has been scanned along the $\mathrm{y}$ direction at $\mathrm{x}=0$ in three different configurations: bare scintillator and with 1 and $2 \mathrm{~mm}$ thick PMMA slab covering a portion of the detector thin window (Fig. 3).

\section{RESULTS}

The output image is a map of digital units (ADU) per pixel: the experimental number of photons can be assessed multiplying the digital units by the gain of the CCD and dividing by the quantum efficiency of the sensor (that is $45 \%$ at $425 \mathrm{~nm}$ ). Then, for comparison with the simulation outputs, the number of photons is summed up along the direction perpendicular to the beam.

Firstly, the plastic scintillator was irradiated with a fixed carbon ion beam of $248.5 \mathrm{MeV} / \mathrm{u}$ to evaluate the quenching factor. The measured integral distribution of the number of photons per CCD pixel was compared with the simulated one (with the semianalytical model). A Birks coefficient of $0.0101 \mathrm{~g} /\left(\mathrm{MeV} \mathrm{cm}^{2}\right)$ was estimated. This value well agrees with those reported in the literature, ${ }^{18}$ for polyviniltoluenebased scintillators.

The data on which the estimation of the Birks coefficient was performed are shown in Fig. 7.

The calculation of the Birks coefficient was performed fitting with a numerical tool the Bragg peak of the experimental data with simulated ones. The figure shows simulated data also for different Birks coefficients, in order to have the sensitivity on how the Birks coefficient modifies the Bragg peak. It should be noticed that the Birks coefficient changes the amplitude of the Bragg peak but has no substantial influence on the beam range evaluation, which is the final goal of the

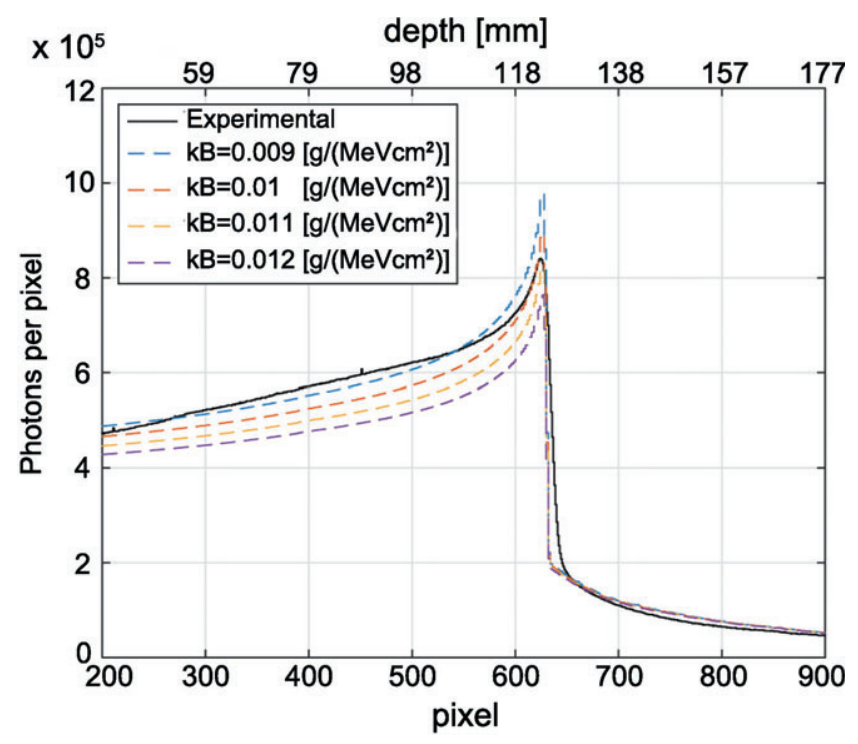

Fig. 7. Comparison between Monte Carlo simulations with different Birks coefficients and experimental data for a $248.5 \mathrm{MeV} / \mathrm{u}$ carbon ion beam impinging on the detector. [Color figure can be viewed at wileyonlinelibra ry.com]

discussed detector. As it can be inferred from Fig. 7, a $20 \%$ variation on the Birks coefficient is well visible in the peak height but does not change by a sensitive amount the Bragg peak position.

Using the estimated quenching factor, simulations were performed with proton beams impinging on the detector. In Figs. 8 and 9, the number of photons per pixel obtained with the two simulation methods is compared with the measured one for proton beams of 81 and $148 \mathrm{MeV}$.

Both numerical models show a rather good agreement with experimental data in terms of detected photons per pixel. The tail in the measured signal after the Bragg peak and other discrepancies has been ascribed to the nonperfect shielding of the ambient light which results in a nonuniform contamination on the acquired image.

Figure 10 shows the results of experimental and Monte Carlo simulations obtained with the superimposition of a proton and a carbon-fragment images. The figure shows the two separated contributions and the sum of the signals. The acquired 2D images before the integration are shown on the left side of Fig. 10 in order to exhibit how real acquired images look like.

Figure 10 shows that the Bragg peak of protons is still visible also in the presence of the light emitted by carbon pertaining fragments. The agreement between simulated and measured light in terms of photons per pixel detected by the sensor is still rather good (i.e., it is within the $20 \%$ in the proton Bragg peak region), except for the region where the beam enters into the scintillator (initial hundreds of pixels). In this region, optical artifacts need to be taken into account, as it will be explained in the following.

The Monte Carlo models just shown can be employed in generating a simulated image of the real future situation: a real mixed helium and carbon beam (10\% of He particles in 


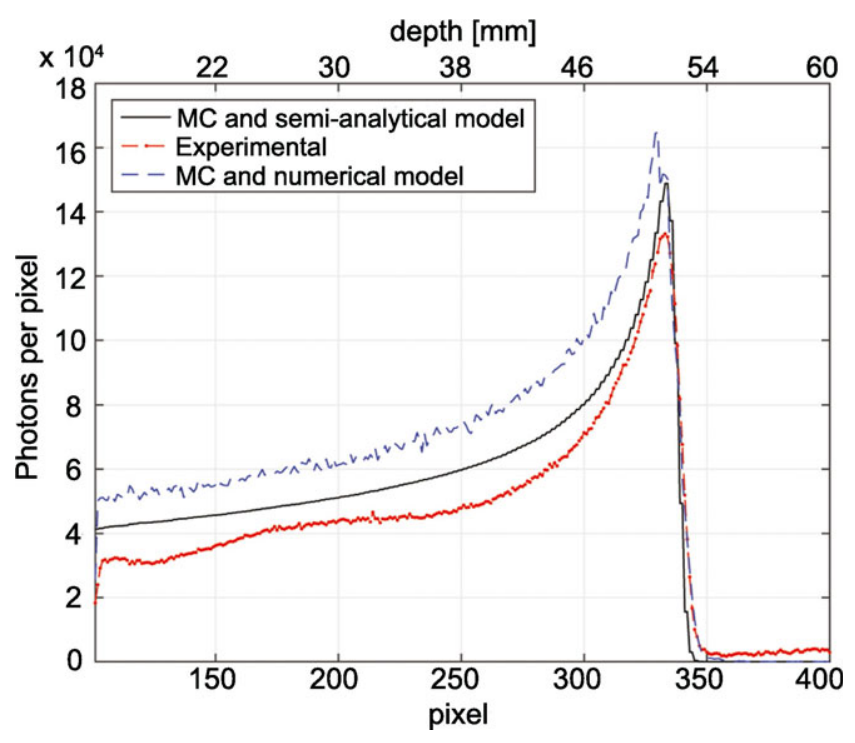

FIG. 8. Comparison between simulated and measured photons per pixel for proton beam of $81 \mathrm{MeV}$. [Color figure can be viewed at wileyonline library.com]

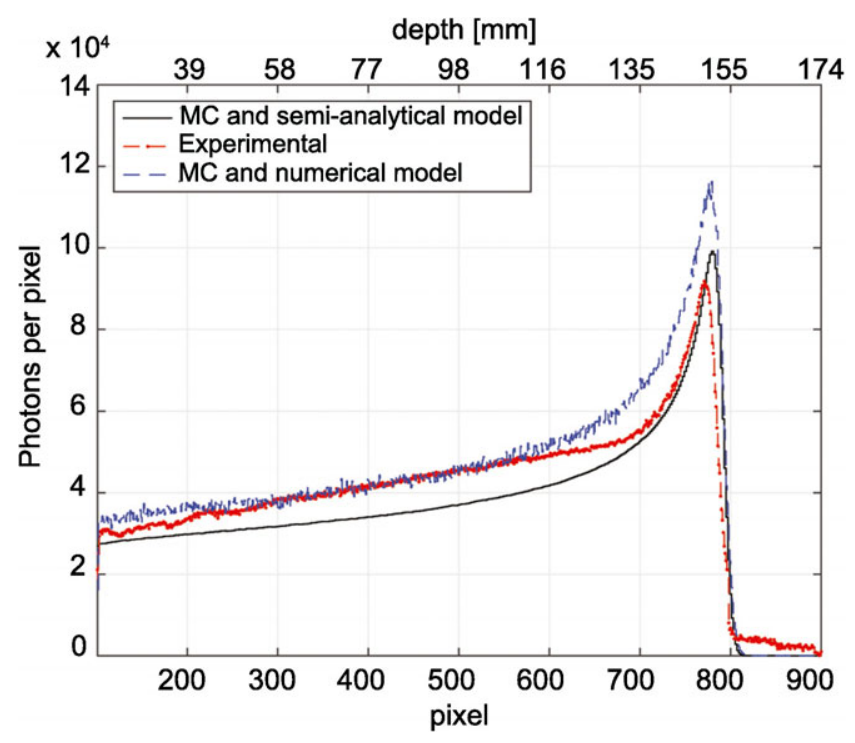

FIG. 9. Comparison between simulated and measured photons per pixel for proton beam of $148 \mathrm{MeV}$. [Color figure can be viewed at wileyonline library.com]

number) impinging on the detector after crossing a $20 \mathrm{~cm}$ PMMA slab. The resulting simulated light (exploiting the analytical model) that would be seen by the sensor is shown in Fig. 11. Figure 11 refers to a $226.5 \mathrm{MeV} / \mathrm{u}$ mixed carbon and helium beam irradiating the detector placed after the $20 \mathrm{~cm}$ slab.

The light referring to the proton case and plotted in Fig. 10 is reported also in Fig. 11 for comparison. As expected, the light emitted by helium is greater than the one emitted by protons but, due to the quenching it is only about two times the protons light emission. Nevertheless, the helium peak appears more distinguishable and narrower with respect to the proton one and, therefore, the simulations highlights that testing the system with protons is conservative with respect to the magnitude of the signal.

It is worth mentioning that the output of the two models is strongly dependent on the values of the parameters, such as refraction index and diaphragm aperture, just to mention some. Therefore, any uncertainty on these parameters affects the simulated light intensity.

Figures 12 and 13 show that for different x positions (i.e., different distances between the light sensor and the irradiated point, see Fig. 11), the particle range in the scintillator appears different even if the energy is the same, while this effect is not present for different $\mathrm{y}$ and same $\mathrm{x}$ irradiations.

This phenomenon is due to three main optical effects: perspective, refraction (the scintillator refraction index is 1.58), and reflection.

The listed optical effects can all be corrected if the position of the beam in the scintillator is known. At least in an initial phase, the beam position will not be measured inside the HeCheck system and will be acquired by the dose delivery system.

The effect of perspective distortion on the measured range can be corrected using:

$$
R_{p}=\left[\frac{K_{x=100}-K_{x=-100}}{L_{s}} x+K_{x=100}\right] \cdot \Delta N
$$

where $\mathrm{K}$ is the physical dimension corresponding to the pixel size and depends on $\mathrm{x}$ and $\Delta \mathrm{N}$ is the number of pixels corresponding to the length on the image of the emitted light.

The range on the image was evaluated by detecting the pixel in correspondence of an emitted light equal to the $50 \%$ of the maximum (i.e., the Bragg peak). In the considered configuration, $K_{x}=100=0.236 \mathrm{~mm} /$ pixel, $\quad K_{x}=-100=0.296 \mathrm{~mm} /$ pixel.

Refraction effects can be fixed if the apparent position of the pixel $x_{a}$ is considered, as shown in Fig. 4. Then, the range becomes a function of $x_{a}$ :

$$
R_{r}=R_{p}\left(x_{a}\right)
$$

where $x_{a}=\left(x-\frac{L_{s}}{2}\right) \frac{n_{a}}{n_{s}}$.

Finally, the reflection of the Bragg peak on the lateral surface of the scintillator yields an additional term on the equation that turns into:

$$
\begin{aligned}
R= & {\left[\frac{K_{x=100}-K_{x=-100}}{L_{s}}\left(x-\frac{L_{s}}{2}\right) \frac{n_{a}}{n_{s}}+K_{x}=100\right] \cdot \Delta N } \\
& -\frac{H_{r}}{L_{s}}\left(x-\frac{L_{s}}{2}\right)
\end{aligned}
$$

where $\mathrm{H}_{\mathrm{r}}$ is a distance taking into account light reflection; $H_{r}=16 \mathrm{~mm}$ in the present setup.

By using such corrections on the acquired light, it is possible to correct the estimated ranges for the nine points irradiated as it is listed into Table I.

The uncorrected ranges in Table I are calculated multiplying $\Delta \mathrm{N}$ by a mean value of $\mathrm{K}$ independent from the position $\mathrm{x}$.

The table shows how the corrections can retrieve an information about the measured range in the nine irradiated 

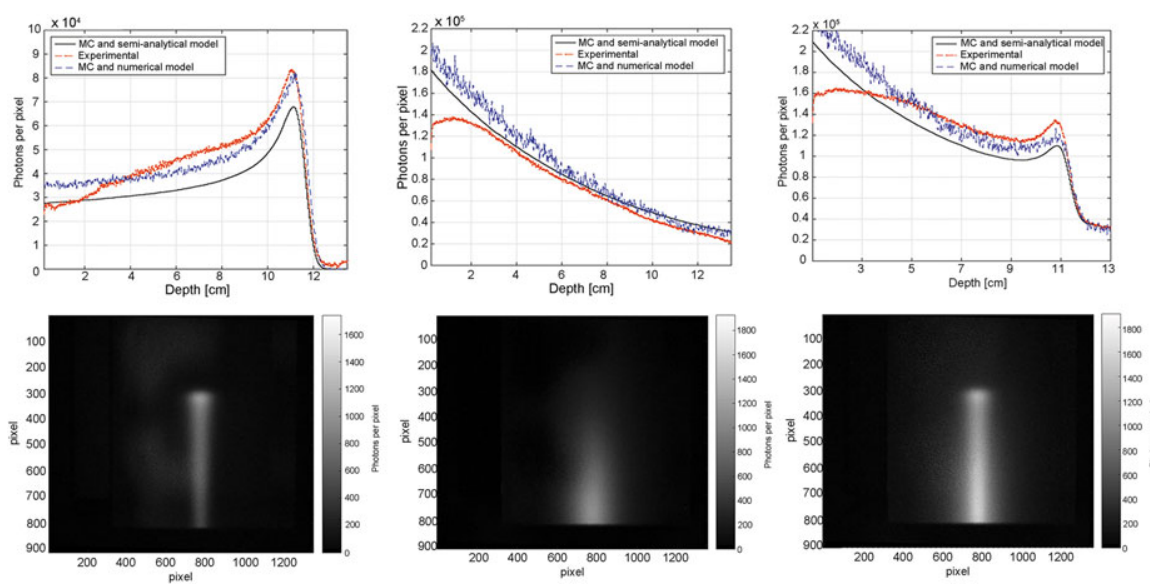

FIG. 10. Comparison between simulated and measured photons per pixel for a $226.9 \mathrm{MeV}$ proton beam and a $226.5 \mathrm{MeV} / \mathrm{u}$ carbon beam. Protons are $10 \%$ in number with respect to carbon ions. The image shows the comparison for only protons (up-left), carbon fragments (up-center), and the superimposed image (up-right). Bottom: 2D acquired images converted in photons per pixel. [Color figure can be viewed at wileyonlinelibrary.com]

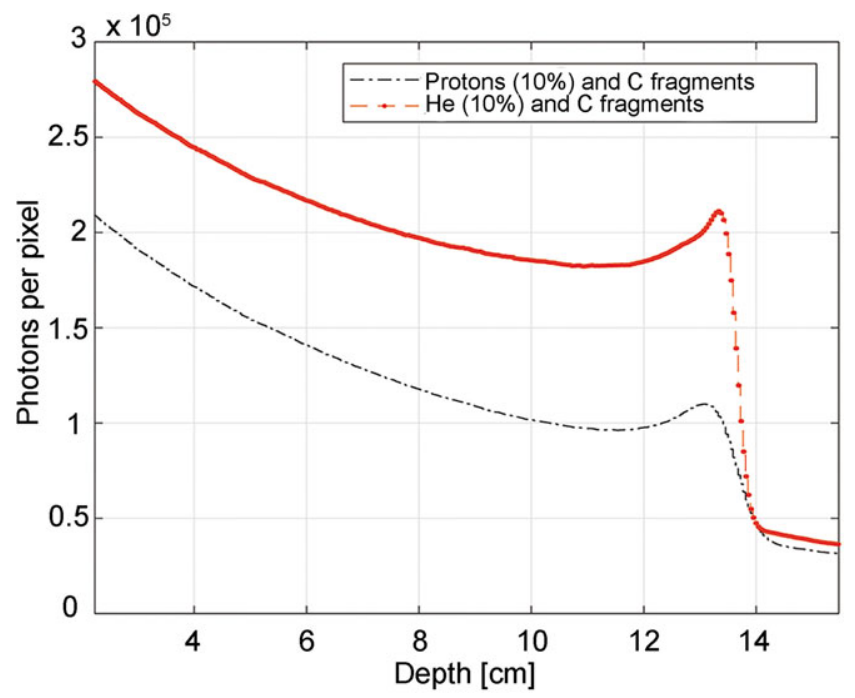

FIG. 11. Simulated photons per pixel for proton beam of $226.5 \mathrm{MeV}$ superimposed to $226.5 \mathrm{MeV} / \mathrm{u}$ carbon ion fragments and a mixed helium and carbon $226.5 \mathrm{MeV} / \mathrm{u}$ beam. The FLUKA simulations employ the analytical model and take into account the quenching of the scintillator. [Color figure can be viewed at wileyonlinelibrary.com]

positions. The ranges for the $81 \mathrm{MeV}$ proton beam in the table are in the confidence range $( \pm 2 \sigma)$ $49.295 \pm 0.234 \mathrm{~mm}$. The measurements were repeated for 118.2 MeV protons (and $198 \mathrm{MeV} / \mathrm{u}$ carbon ions) obtaining values laying in the interval $99.469 \pm 0.304 \mathrm{~mm}$.

Finally, the recorded images placing different slabs before the detector thin window are shown in Fig. 14. Looking at the images, it is easily distinguishable were the $1 \mathrm{~mm}$ or $2 \mathrm{~mm}$ slab is. For a quantitative comparison between the profiles, three Bragg peaks are shown in Fig. 15. The profiles in Fig. 15 refer to the same scan position but to different slabs before the detector thin window. The present resolution of the system is sufficient to detect and distinguish the PMMA slabs. In fact, sampling the light in 15 positions along the scan of the beam, it is possible to evaluate the corresponding ranges plotted in the lower panel of Fig. 14.

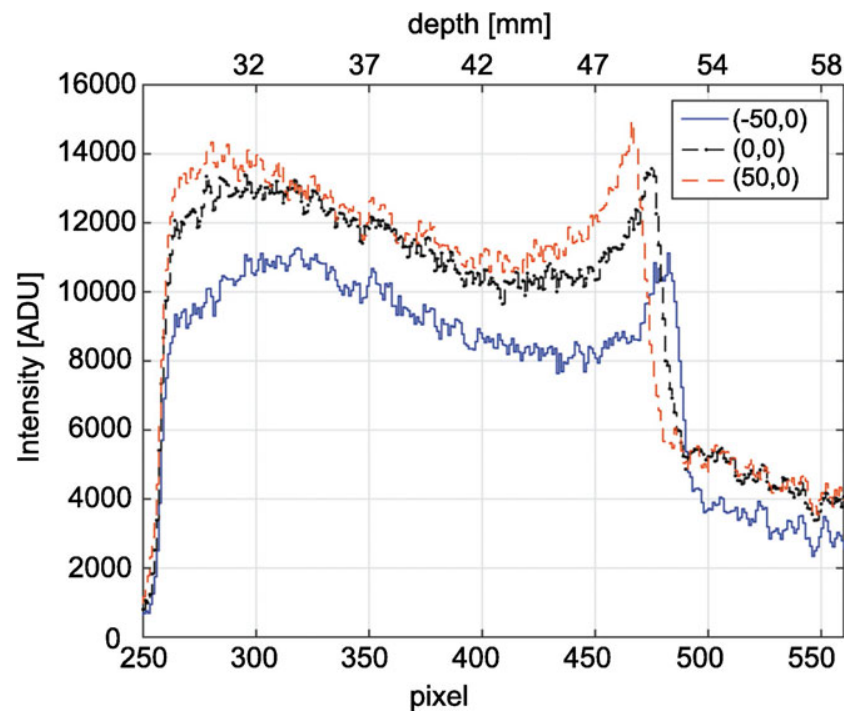

FIG. 12. Light collected for different irradiated points having the same vertical coordinate using $81 \mathrm{MeV}$ protons and $172.4 \mathrm{MeV} / \mathrm{u}$ for carbon ions. [Color figure can be viewed at wileyonlinelibrary.com]

\section{DISCUSSION}

The results of the previous section show that the proposed system, composed by a scintillator and a CCD camera, is able to measure the range of a proton beam also in the presence of the noise produced by carbon ion fragments. The number of protons was one tenth of the carbon ion one.

Although experiments with helium were not carried out, being $\mathrm{He}$ not available at CNAO, we dare to conclude the admixture of helium in a carbon ion beam could be useful for an online verification of the correctness of the treated volume during a treatment session. In fact, the helium Bragg peak signal is expected to be more clearly distinguishable from Cion fragments than the proton one, using the same fraction in number. In fact, employing the developed Monte Carlo models a simulation of the real situation (i.e., mixed helium and 


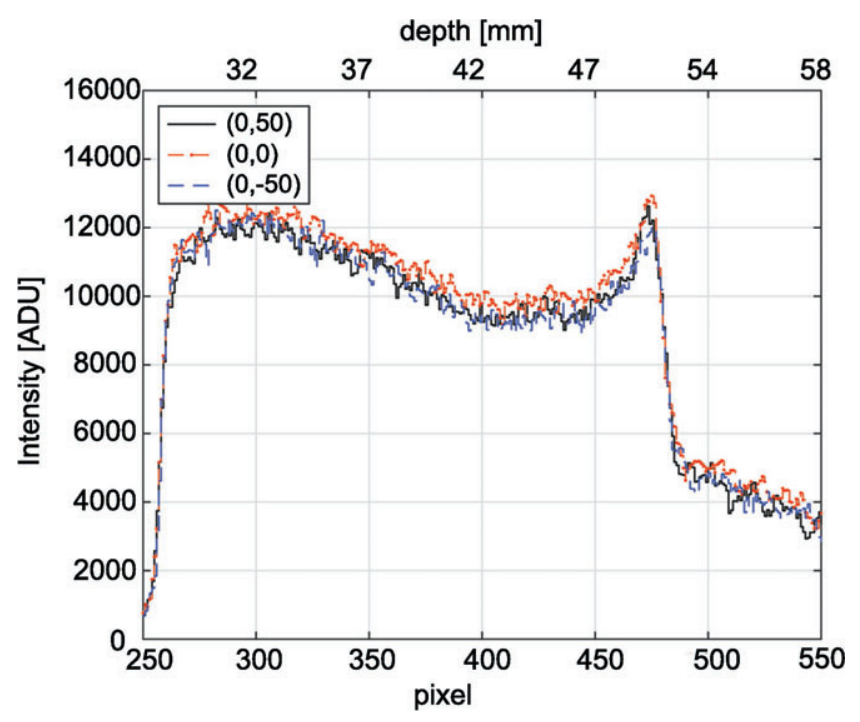

FIG. 13. Light collected for different irradiated points having the same horizontal coordinate using $81 \mathrm{MeV}$ protons and $172.4 \mathrm{MeV} / \mathrm{u}$ for carbon ions. [Color figure can be viewed at wileyonlinelibrary.com]

TABLE I. Estimated ranges before and after the correction for a $81 \mathrm{MeV}$ proton beam.

\begin{tabular}{lrcc}
\hline \hline $\mathrm{x}[\mathrm{mm}]$ & $\mathrm{y}[\mathrm{mm}]$ & Uncorrected range $[\mathrm{mm}]$ & Corrected range $[\mathrm{mm}]$ \\
\hline-50 & 50 & 54.542 & 49.417 \\
-50 & 0 & 54.542 & 49.417 \\
-50 & -50 & 54.306 & 49.151 \\
0 & 50 & 52.889 & 49.333 \\
0 & 0 & 52.889 & 49.333 \\
0 & -50 & 52.889 & 49.333 \\
50 & 50 & 51.236 & 49.389 \\
50 & 0 & 51.000 & 49.143 \\
50 & -50 & 51.000 & 49.143 \\
\hline \hline
\end{tabular}

carbon beam of the same energy per nucleon) showed a steeper and more distinguishable helium Bragg peak with respect to protons in spite of the higher quenching into the scintillator (employing the same number of particles).

In this paper, the general framework and some instruments (i.e., Monte Carlo tools) that could be useful for future development were discussed.

Despite these results, in order to have a real working system, useful during a treatment, many other issues have to be addressed.

At this point of the research, the detector has no lateral resolution with respect to the beam direction. The irradiated position has to be known a priori and is provided by the dose delivery system. Assuming a straight path of the beam into the patient, the $2 \mathrm{D}$ map of the residual range has a spatial resolution equal to the dose delivery system one. For the implementation of such a system in a more complex scanned irradiation, image acquisition and processing have to be fast enough to give range information for each irradiation position. Conceptually, the processed image of the scintillation light will look like a single pencil beam like the data shown in this paper. In this
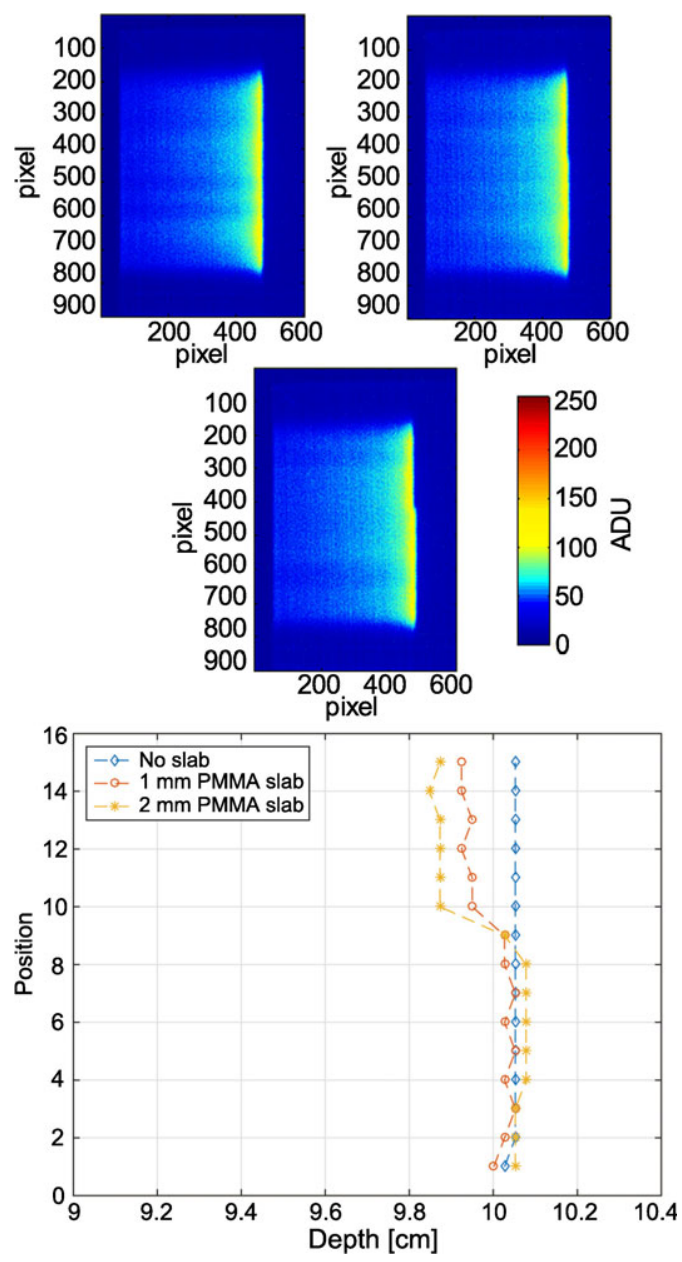

FIG. 14. Upper panel: images of a scanning of a proton beam along the direction y. Top left bare scintillator, top right with $1 \mathrm{~mm}$ slab and bottom with $2 \mathrm{~mm}$ slab. Lower panel: measured ranges for each scanned position. [Color figure can be viewed at wileyonlinelibrary.com]

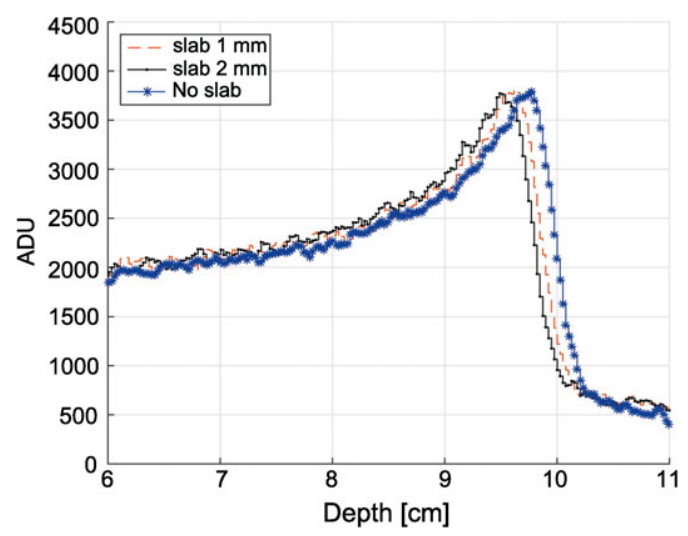

FIG. 15. Profiles in correspondence of position 3 placing $1 \mathrm{~mm}, 2 \mathrm{~mm}$, or no PMMA slab in front of the thin window of the detector. [Color figure can be viewed at wileyonlinelibrary.com]

way, artifacts like range mixing and superimposition of different signals could be reduced. In any case, in the real situation the crossed tissue will be the patient: range mixing could be present also for single pencil beam. This issue could be taken into account by comparing the output image with reference 
images obtained with Monte Carlo simulations of the real nonhomogenous case using the numerical tools presented in this paper. This issue certainly deserves further investigations and will be matter of future work.

Future progresses will be devoted to the development of such fast acquisition and elaboration system based on a scintillator and an optical sensor.

Other issues related to helium acceleration in the synchrotron and the different helium scintillation quenching with respect to protons are not discussed here and will be matter of future work.

\section{CONCLUSIONS}

Since, at same energy per nucleon, the range of ${ }^{4} \mathrm{He}$ is approximately three times the range of ${ }^{12} \mathrm{C}$, but the magnetic rigidity is very similar, a mixed particle beam can be addressed to the patient; thus, treatment and confirmation of patient thickness can be carried out simultaneously. FLUKA simulations show that, adding $10 \%$ of helium ions to a carbon beam, the dose contribution due to helium is approximately $1 \%$ of the dose deposited by the carbon in the SOBP.

To measure the residual range of helium emerging from the target volume, a prototype system, based on a plastic scintillator and a CCD camera, has been built. Two different Monte Carlo models of the optical system have been implemented to evaluate the light emitted by the scintillator.

Being helium not available at $\mathrm{CNAO}$, the models have been validated by measuring the light produced by a proton beam impinging on the detector and by the fragments produced by a carbon beam in a water equivalent slab located just upstream the scintillator.

The numerical predictions of both the models are shown to reproduce well the shape (within the $20 \%$ in absolute terms) of the measurements in terms of emitted light signal.

We have measured the range of $81-118.2 \mathrm{MeV}$ protons with a resolution of $\pm 0.234 \mathrm{~mm}$ and $\pm 0.304 \mathrm{~mm}$, respectively. Furthermore, we verified that the system is able to detect discrepancies in the expected range with a resolution better than $1 \mathrm{~mm}$.

Although many other technical issues have to be addressed for a real implementation in a clinical environment, these preliminary results are extremely promising and suggest that a surrogate of real-time verification of the beam range into the patient during a treatment with carbon ions is possible with this technique.

\section{ACKNOWLEDGMENT}

The authors thank Mr. Giuliano Monti (Tecnosky srl) for providing part of the equipment of the experimental setup.

\section{CONFLICTS OF INTEREST}

The authors have no relevant conflicts of interest to disclose.

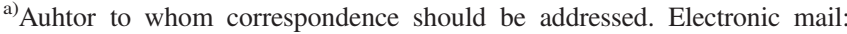
davide.mazzucconi@polimi.it.
}

\section{REFERENCES}

1. Durante M, Orecchia R, Loeffler JS. Charged-particle therapy in cancer: clinical uses and future perspectives. Nat Rev. 2017;14:483-495.

2. Jäkel O, Hartmann GH, Karger CP, Heeg P, Rassow J. Quality assurance for a treatment planning system in scanned ion beam therapy. Med Phys. 2000;27:1588-1600.

3. Enghardt W, Parodi K, Crespo P, Fiedler F, Pawelke J, Pönisch F. Dose quantification from in-beam positron emission tomography. Radiother Oncol. 2004;73:S96-S98.

4. Enghardt W, Debus J, Haberer T, et al. The application of PET to quality assurance of heavy-ion tumor therapy. Strahlenther Onkol. 1999;175 (Suppl 2):33-36.

5. Knopf AC, Lomax A. In vivo proton range verification: a review. Phys Med Biol. 2013;58:R131.

6. Gianoli C, De Bernardi E, Ricotti R, et al. First clinical investigation of a 4D maximum likelihood reconstruction for 4D PET-based treatment verification in ion beam therapy. Radiother Oncol. 2017;123:339345.

7. Richter C, Pausch G, Barczyk S, et al. First clinical application of a prompt gamma based in vivo proton range verification system. Radiother Oncol. 2016;118:232-237.

8. Muraro S, Battistoni G, Collamati F, et al. Monitoring of hadrontherapy treatments by means of charged particle detection. Front Oncol. 2016;6:177.

9. Parodi K. Heavy ion radiography and tomography. Physica Med. 2014;30:539-543.

10. Knoll GF. Radiation Detection and Measurement. Hoboken, NY: Wiley and sons; 2010.

11. Böhlen TT, Cerutti F, Chin MPW, et al. The FLUKA Code: developments and challenges for high energy and medical applications. Nucl Data Sheets. 2014;120:211-214.

12. Ferrari A, Sala PR, Fassò A, Ranft J. FLUKA: a multi-particle transport code. CERN-2005-10; INFN/TC_05/11, SLAC-R-773. 2005.

13. Pullia M. Synchrotrons for Hadrontherapy. In Reviews of Accelerator Science and Technology. 2009;2:157-178.

14. Beddar S, Archambault L, Sahoo N, et al. Exploration of the potential of liquid scintillators for real-time 3D dosimetry of intensity modulated proton beams. Med Phys. 2009;36:1736-1743.

15. Archambault L, Poenisch F, Sahoo N, et al. Verification of proton range, position, and intensity in IMPT with a 3D liquid scintillator detector system. Med Phys. 2012;39:1239-1246.

16. Poenisch F, Archambault L, Briere TM, et al. Liquid scintillator for 2D dosimetry for high-energy photon beams. Med Phys. 2009;36:14781485.

17. Yu T, Boone JM. Lens coupling efficiency: derivation and application under differing geometrical assumptions. Med Phys. 1997;24: $565-570$.

18. Wang LLW, Perles AL, Archambault L, Sahoo N, Mirkovic D, Beddar $\mathrm{S}$. Determination of the quenching correction factors for plastic scintillation detectors in therapeutic high-energy proton beams. Phys Med Biol. 2012;57:7767. 